\title{
A Miniaturized Neuroprosthesis Suitable for Implantation Into the Brain
}

\author{
Mohammad Mojarradi, Member, IEEE, David Binkley, Senior Member, IEEE, Benjamin Blalock, Member, IEEE, \\ Richard Andersen, Norbert Ulshoefer, Member, IEEE, Travis Johnson, Member, IEEE, and Linda Del Castillo
}

\begin{abstract}
This paper presents current research on a miniaturized neuroprosthesis suitable for implantation into the brain. The prosthesis is a heterogeneous integration of a 100-element microelectromechanical system (MEMS) electrode array, front-end complementary metal-oxide-semiconductor (CMOS) integrated circuit for neural signal preamplification, filtering, multiplexing and analog-to-digital conversion, and a second CMOS integrated circuit for wireless transmission of neural data and conditioning of wireless power. The prosthesis is intended for applications where neural signals are processed and decoded to permit the control of artificial or paralyzed limbs. This research, if successful, will allow implantation of the electronics into the brain, or subcutaneously on the skull, and eliminate all external signal and power wiring. The neuroprosthetic system design has strict size and power constraints with each of the front-end preamplifier channels fitting within the $400 \times 400-\mu \mathrm{m}$ pitch of the 100-element MEMS electrode array and power dissipation resulting in less than a $1^{\circ} \mathrm{C}$ temperature rise for the surrounding brain tissue. We describe the measured performance of initial micropower low-noise CMOS preamplifiers for the neuroprosthetic.
\end{abstract}

Index Terms-Bio-MEMS, brain, complementary metal-oxidesemiconductor (CMOS) micropower, computer interface, cortical signals, heterogeneous integration, integrated-circuit electronics, low-noise preamplifiers, microelectromechanical system (MEMS) packaging, microelectromechanical system (MEMS) probe, neuroprosthesis, neuroprosthetic devices.

\section{INTRODUCTION}

$\mathbf{P}$ ASSIVE microelectrode arrays have been widely used by researchers as a neuroprosthetic tool to extract electrical signals from the brain. The microelectrode arrays are directly connected to measurement instruments through a large bundle of wires and are placed into the brain using surgical techniques. The large number of wires connected to current passive microelectrode arrays limits their widespread use as permanent

Manuscript received July 10, 2002; revised March 25, 2003. This work was supported under a contract with the Defense Advanced Research Projects Agency for the California Institute of Technology.

M. Mojarradi, T. Johnson, and L. Del Castillo are with NASA Jet Propulsion Laboratory, Pasadena, CA 91109 USA (e-mail: Mojarradi@jpl.nasa.gov; Travis.W.Johnson@jpl.nasa.gov; Linda.Y.Delcastillo@jpl.nasa.gov).

D. Binkley is with the Electrical and Computer Engineering Department, University of North Carolina at Charlotte, Charlotte, NC 28223 USA (e-mail: dmbinkle@uncc.edu; Norbert.Ulshoefer@gmx.de).

B. Blalock is with the Electrical and Computer Engineering Department, University of Tennessee, Knoxville, TN 37996 USA (e-mail: bblalock@ece.utk.edu).

R. Andersen is with the Division of Biology, California Institute of Technology, Pasadena, CA 91125 USA (e-mail: Andersen@vis.caltech.edu).

N. Ulshoefer was with the University of North Carolina at Charlotte, Charlotte, NC 28223 USA. He is now with Infineon Technologies AG, Germany (e-mail: Norbert.Ulshoefer@gmx.de).

Digital Object Identifier 10.1109/TNSRE.2003.810431 neuroprosthetic devices. In addition, there is an increasing demand for deploying microelectronics to develop a more sophisticated generation of neuroprosthetic devices capable of producing high-quality electrical signals while significantly reducing the number of connection wires or potentially eliminating the wires entirely using a wireless data link.

A neuroprosthetic implant, such as the one described in this work or reported in [1]-[3], might ultimately control an artificial human prosthesis for paralyzed patients. Such a neuroprosthetic implant would amplify and decode cortical signals in order to control an artificial limb or other external device. Simple visual observation of the performance of the prosthetic system by the patient may close the loop between the artificial prosthesis and the brain.

The development of electronics for the cortical recording is done as a part of a collaborative research program to develop a neuroprosthetic for paralyzed patients [4]. Neurophysiological investigation focuses on cortical recording and evaluation of action planning signals from the posterior parietal cortex (PPC) of monkeys. This area of the cortex may have advantages for applications in neuroprosthetics research. The visual-motor areas of the PPC appear to be very adaptive and able to quickly learn new representations. The parietal reach region (PRR) of the PPC may show little degeneration with paralysis since it provides only modest projections to the spinal cord, and its major afferents appear to be visual, rather than somatosensory. The signals from this region are believed to indicate movement intentions and can provide a source for decoding high-level, cognitive plans for actions. The neurophysiology laboratory has successfully demonstrated real time decoding of neural signals from the PRR. In these closed-loop experiments, monkeys were trained to position a cursor on a computer screen without making an actual reach movement. The cells showed remarkable plasticity in the closed-loop condition, with approximately half of them significantly increasing their direction tuning within 10 to 50 trials when the animals were using the neural activity to position the cursor [5].

\section{HETEROGENEOUS INTEGRATION OF NEUROPROSTHETIC IMPLANT}

Heterogeneous integration of commercially available passive Microeelectromechanical system (MEMS)-based electrode arrays with the associated electronic circuitry required the development of bio-MEMS packaging technology. Some specific challenges of this integration include 1) the handling of the MEMS array in a way that allows reliable bonding of the fine 
$100-\mu \mathrm{m}$ joints without damaging the delicate structures and 2) the selection of underfills and coatings capable of isolating the electronics from the body while maintaining biocompatibility. Fig. 1 shows the heterogeneous integration of the neuroprosthetic implant under development, consisting of the MEMS brain probe, integrated complementary metal-oxide-semiconductor (CMOS) electronics and wireless transceiver and power conditioning integrated circuit. The MEMS intracortical electrode array [6] consists of a $10 \times 10$ matrix $(400 \mu \mathrm{m}$ pitch) of fine, brittle 1-mm-long electrodes. The electrodes are fabricated from $\mathrm{Si}$, which is known to be compatible with brain tissue [7], isolated from one another by rows of $\mathrm{SiO}_{2}$ and jacketed with SiN. Platinum $(\mathrm{Pt})$ at the tips of the electrodes allows for signal transmission. It should be noted that the chip we have developed can be used with other electrode technologies, such as microwires and tetrodes with simple modification of the connections between the electrode arrays and the chip.

Area array flip-chip technology is used to attach the electronic chip and the MEMS device. The motivations for using flip-chip technology were based on miniaturization to obtain electrical performance improvements and accommodating interconnect density demands. The delicate nature of the Si electrodes complicates the design of the holding fixture. Fragile ledges inhibited the use of mechanical fixtures, and processing temperatures reduced the number of viable adhesives. Through process development, a holding fixture was designed with a cavity that was slightly deeper than the arrays. The fixture incorporated a "shelf" along the edges, which protected the ledges and ensured a relatively even bonding surface. In addition to fixture design and fabrication, the method of array placement within the holding fixtures influenced the quality of the interconnect. Joining processes were investigated using the MEMS electrode arrays and metallized substrates (in place of the electronic chip) [8]. Bonding was performed using both conductive epoxy (FDA approved) and solder. Two heterogeneously integrated neuroprosthetics implants are exhibited in Figs. 1 and 2.

The need for biocompatibility dominated the search for underfills and coatings. However, the fine gap (only 50-75 $\mu \mathrm{m}$ ) between the bonded chip and array required the use of a low-viscosity underfill. Once it was proven to completely fill the gap between the electrode array and the substrate without influencing the solder connection, a low-viscosity biocompatible epoxy was chosen as the underfill. After underfilling, the entire assembly was coated with Parylene $\mathrm{C}$ because of its low absorption of water and capability to conformally coat, as well as the fact that it has been used in medical implants for some time. It should be noted that the array tips cannot be coated and were thus held in polyethylene glycol (PEG) during the coating procedure. To ensure the function and form of the commercially purchased electrode array as well as that of the fully packaged device, a thorough well-defined incoming/outgoing inspection procedure was developed.

\section{FRONT-END CMOS INTEGRATED CirCUIT}

Fig. 3 shows a block diagram of the front-end $0.5-\mu \mathrm{m}$ CMOS integrated circuit under development for neural signal

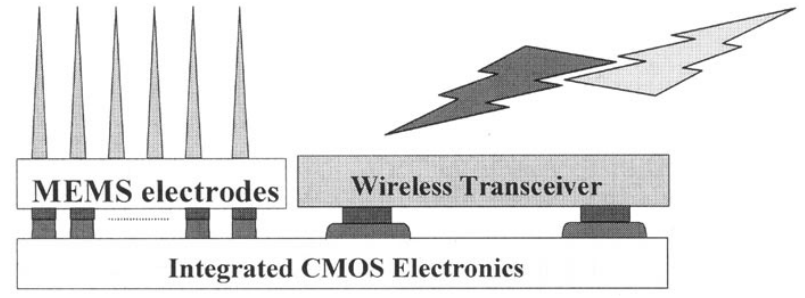

Fig. 1. Heterogeneous integration of neuroprosthetic implant consisting of MEMS probe, integrated front-end CMOS electronics, and wireless transceiver.

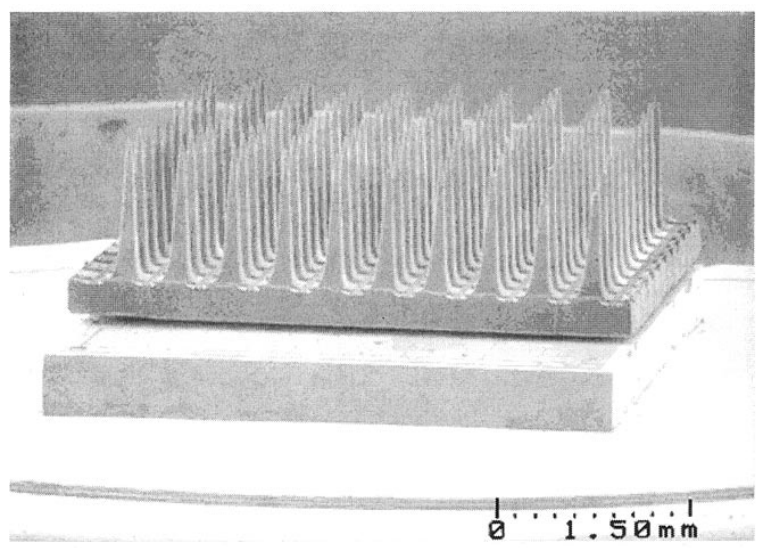

Fig. 2. Scanning electron microscope (SEM) image of a heterogeneously integrated MEMS electrode array and electronic chip.

amplification, filtering, multiplexing, dc offset correction, and analog-to-digital (A/D) conversion. An architecture containing an individual preamplifier for each electrode array element was selected to minimize charge injection associated with input multiplexing into a common preamplifier. Charge injection associated with metal-oxide-semiconductor (MOS) transistor switching at the electrode array could significantly disturb adjacent electrode signals as charge is injected back into the brain resistance and capacitance. If charge injection is significantly high, there is also the potential of brain activation.

As shown in Fig. 3, multiplexing will be performed at the output of the front-end preamplifiers, which provides a high degree of charge injection isolation from the multiplexer. Since the preamplifier voltage gains are feedback regulated at typically $80 \mathrm{~V} / \mathrm{V}$ (the gains are programmable for most of the preamplifiers considered), the presence of MOS device dc mismatch and brain dc offset potentials requires a dc offset subtraction at the multiplexing input circuit. This will be done using a digital-toanalog (D/A) converter to provide the unique correction value for each preamplifier input channel and will be dynamically updated. The dynamic updates from the offset correction circuits eliminate the need for the traditional high-pass filters with very low cutoff frequency for the preamplifiers and enhance the noise performance of the circuit. Following dc offset correction, additional voltage gain will be provided before (A/D) conversion of the conditioned neural signals.

The use of individual electrode array preamplifiers, while minimizing the problems associated with multiplexing charge injection back into the brain, complicates the preamplifier design because of the size constraints associated with 100 pream- 


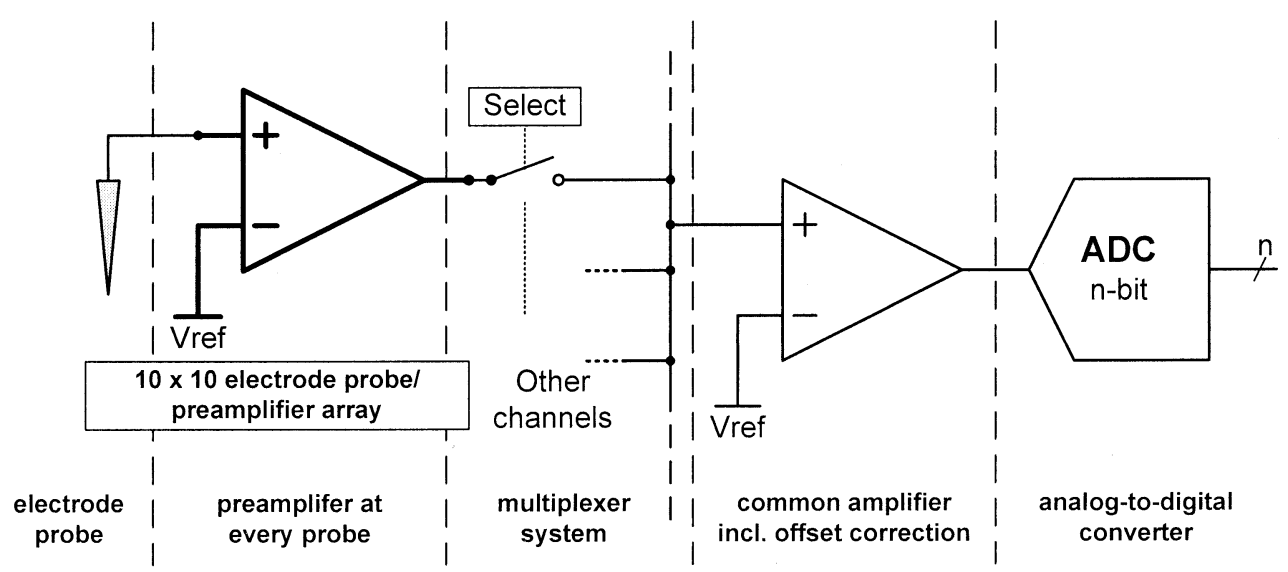

Fig. 3. Block diagram of front-end 0.5- $\mu \mathrm{m} \mathrm{CMOS} \mathrm{integrated} \mathrm{circuit} \mathrm{for} \mathrm{neural} \mathrm{signal} \mathrm{amplification,} \mathrm{filtering,} \mathrm{multiplexing,} \mathrm{and} \mathrm{analog-to-digital} \mathrm{(A/D)} \mathrm{conversion.}$

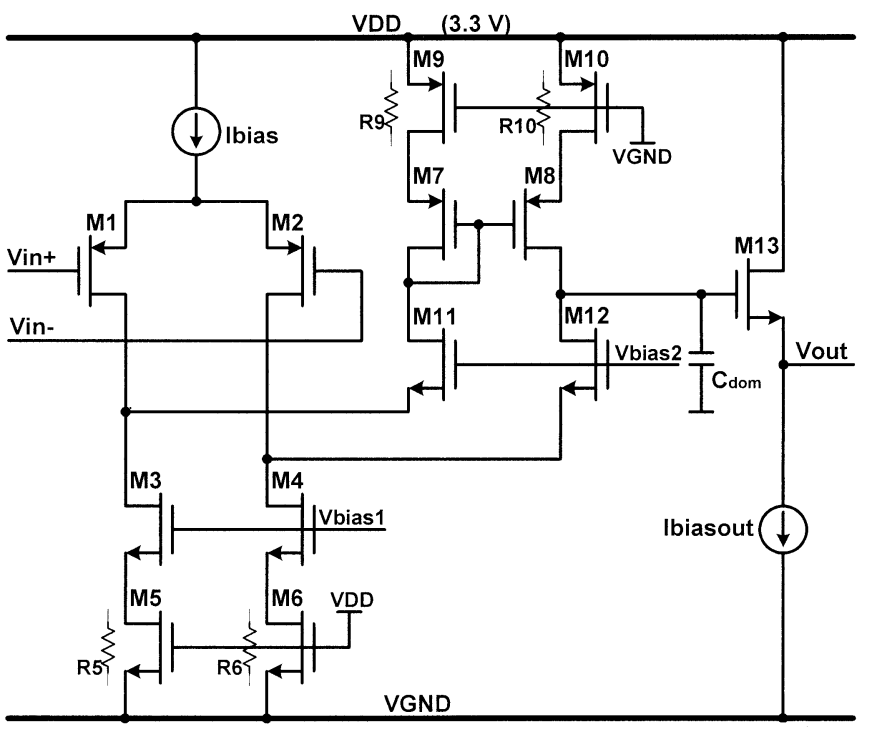

Fig. 4. Abbreviated schematic of micropower low-noise CMOS preamplifier for amplifying each intracortical electrode signal.

plifiers fitted directly behind the $400 \times 400-\mu \mathrm{m}$ pitch electrode array. In addition, neuroprosthetic implant power consumption must be limited to prevent a brain tissue temperature rise of over $1{ }^{\circ} \mathrm{C}$, which could have long-term effects on the brain. As a result, power efficient micropower low-noise CMOS preamplifiers are critical for the success of this research.

Fig. 4 shows an abbreviated schematic of the micropower, low-noise CMOS preamplifier under development. metal-oxide-semiconductor field-effect transistors (MOSFETs) M1 and M2 comprise a differential pair using large-area p-type metal-oxide-semiconductor (PMOS) devices to minimize low-frequency flicker noise. Signal current from M1 and M2 is routed through the folded cascode configuration of M11 and M12 to the current mirror provided by M7 and M8 that utilizes resistive noise degeneration provided by M9 and M10. High open-loop gain is developed across the compensation capacitor $C_{\mathrm{DOM}}$ prior to output voltage buffering provided by source follower M13. M3 and M4 are current sources with resistive noise degeneration provided by M5 and M6. Resistive degeneration is utilized to minimize both white and flicker noise of noninput stages. Considerable attention has been paid to ensuring the input pair devices M1 and M2 dominate both white noise and low frequency flicker noise. Low-frequency flicker noise is of considerable interest because of the low-frequency content of neural signals. Input devices M1 and M2 are operated between weak and moderate inversion to ensure high transconductance efficiency $\left(g_{m} / I_{D}\right)$ for low input-referred gate noise voltage at the minimum possible bias current. Resistive preamplifier negative feedback, utilizing deep-ohmic PMOS devices, is used for regulating preamplifier voltage gain.

Table I gives the measured noise performance and power dissipation for a $0.35-\mu \mathrm{m}$ partially depleted silicon-on-insulator (PDSOI) preamplifier used in a proof-of-concept front-end integrated circuit. This integrated circuit contains 64 preamplifiers to support an $8 \times 8$-element intracortical electrode array followed by output multiplexing. This integrated circuit will be tested with an intracortical electrode array in laboratory animals to provide guidance on design closure for the final front-end integrated circuit. In addition, Table I illustrates the Simulation Program with Integrated Circuit Emphasis (SPICE) simulated performance of various $0.5-\mu \mathrm{m}$ bulk CMOS preamplifiers under development, including both a fixed power and a programmable power design. The selection of various power levels is intended to permit the optimum balance of power dissipation and required noise performance. The preamplifier power dissipations given in Table I exclude bias reference circuits that can be common to all 100 preamplifier channels.

\section{Wireless TRANSCEIVER AND WIRELESS POWER INTEGRATED CIRCUIT}

Although an intracortical electrode array and $8 \times 8$-element concept front-end integrated circuit have been fabricated for initial evaluations, the wireless transceiver and wireless power integrated circuit is in the early concept stages. Key issues here will be transmitting digital neural information at the required 
TABLE I

InTRACORTICAl ELECTRODE ARRAY CMOS PREAMPLIFIER PERFORMANCE

\begin{tabular}{|c|c|c|c|c|c|c|}
\hline $\begin{array}{l}\text { CMOS } \\
\text { process }\end{array}$ & $\begin{array}{c}\text { Power per } \\
\text { channel }\end{array}$ & $\begin{array}{c}\text { Total input } \\
\text { noise }^{\mathrm{a}}\end{array}$ & $\begin{array}{c}\text { Input noise } \\
\text { at } 1 \mathrm{~Hz}\end{array}$ & $\begin{array}{l}\text { Input white } \\
\text { noise floor }\end{array}$ & $\begin{array}{l}1 / \text { f corner } \\
\text { frequency }\end{array}$ & Notes \\
\hline $\begin{array}{l}0.35-\mu \mathrm{m} \\
\text { PDSOI } \\
\text { CMOS }\end{array}$ & $\begin{array}{l}9.9 \mu \mathrm{W} \text { at } \\
3.3 \mathrm{~V}\end{array}$ & $\begin{array}{l}8.2 \mu \mathrm{Vrms} \\
\text { (measured) }\end{array}$ & $\begin{array}{c}300 \mathrm{nV} / \sqrt{\mathrm{Hz}} \\
\text { (measured) }\end{array}$ & $\begin{array}{l}65 \mathrm{nV} / \sqrt{\mathrm{Hz}} \\
\text { (measured) }\end{array}$ & $\begin{array}{c}8 \mathrm{~Hz} \\
\text { (measured) }\end{array}$ & $\begin{array}{c}1 \mu \mathrm{A} \text { pair current, } \mathrm{A}_{\mathrm{V}} \\
=49 \mathrm{~V} / \mathrm{V}, \text { fixed } \\
\text { power }\end{array}$ \\
\hline \multirow[t]{4}{*}{$\begin{array}{c}0.5-\mu \mathrm{m} \\
\text { bulk CMOS }\end{array}$} & $\begin{array}{c}60 \mu \mathrm{W} \text { at } \\
5 \mathrm{~V}\end{array}$ & $3.9 \mu \mathrm{Vrms}$ & $170 \mathrm{nV} / \sqrt{ } \mathrm{Hz}$ & $30 \mathrm{nV} / \sqrt{ } \mathrm{Hz}$ & $45 \mathrm{~Hz}$ & $\begin{array}{c}4 \mu \mathrm{A} \text { pair current, } \mathrm{A}_{\mathrm{V}} \\
=81 \mathrm{~V} / \mathrm{V}, \text { fixed } \\
\text { power }\end{array}$ \\
\hline & $\begin{array}{c}15 \mu \mathrm{W} \text { at } 5 \\
\mathrm{~V}\end{array}$ & $8.7 \mu \mathrm{Vrms}$ & $185 \mathrm{nV} / \sqrt{\mathrm{Hz}}$ & $67 \mathrm{nV} / \sqrt{\mathrm{Hz}}$ & $10 \mathrm{~Hz}$ & $\begin{array}{c}1 \mu \mathrm{A} \text { pair current, } \mathrm{A}_{\mathrm{V}} \\
=81 \mathrm{~V} / \mathrm{V}, \text { prog. } \\
\text { power }\end{array}$ \\
\hline & $\begin{array}{c}60 \mu \mathrm{W} \text { at } 5 \\
\mathrm{~V}\end{array}$ & $4.5 \mu \mathrm{Vrms}$ & $171 \mathrm{nV} / \sqrt{ } \mathrm{Hz}$ & $33 \mathrm{nV} / \sqrt{ } \mathrm{Hz}$ & $33 \mathrm{~Hz}$ & $\begin{array}{c}4 \mu \mathrm{A} \text { pair current, } \mathrm{A}_{\mathrm{V}} \\
=81 \mathrm{~V} / \mathrm{V}, \text { prog. } \\
\text { power }\end{array}$ \\
\hline & $\begin{array}{c}240 \mu \mathrm{W} \text { at } 5 \\
\mathrm{~V}\end{array}$ & $2.5 \mu \mathrm{Vrms}$ & $160 \mathrm{nV} / \sqrt{ } \mathrm{Hz}$ & $17 \mathrm{nV} / \sqrt{ } \mathrm{Hz}$ & $100 \mathrm{~Hz}$ & $\begin{array}{c}16 \mu \mathrm{A} \text { pair current, } \\
\mathrm{A}_{\mathrm{V}}=81 \mathrm{~V} / \mathrm{V}, \text { prog. } \\
\text { power }\end{array}$ \\
\hline
\end{tabular}

${ }^{\mathrm{a}}$ Noise integrated over a bandwidth of $1 \mathrm{~Hz}$ to $10 \mathrm{kHz}$.

rate and minimizing coupled energy associated with wireless transmission of power while ensuring less than a $1^{\circ} \mathrm{C}$ temperature rise in neighboring brain tissue.

\section{CONCLUSION}

Current research on a neuroprosthetic implant containing a 100-element MEMS electrode array integrated with CMOS electronics has been presented here. The prosthesis is intended for applications where neural signals are processed and decoded to permit the control of artificial or paralyzed limbs. Flip-chip technology was chosen for the integration of the electronics and MEMS electrode array to accommodate the high density of interconnections. The underfills and coatings chosen for the flip-chip design as well as the Si-fabricated electrodes are biocompatible with brain tissue. An array of CMOS preamplifiers was designed and evaluated for neural signal preamplification at micropower levels necessary to ensure less than $1{ }^{\circ} \mathrm{C}$ temperature rise in surrounding brain tissue. Research is ongoing for filtering, multiplexing, and data conversion of neural signals with wireless transmission to eliminate all external wiring.

\section{ACKNOWLEDGMENT}

The authors acknowledge the assistance of D. H. Ihme and C. E. Hopper of the University of North Carolina at Charlotte for their assistance in preamplifier measurements and preparing this paper.

\section{REFERENCES}

[1] M. A. L. Nicolelis, "Actions from thoughts," Nature, vol. 409, Jan. 2001.

[2] M. Chicurel, "Windows on the brain," Nature, vol. 412, July 2001.

[3] Q. Bai and K. D. Wise, "Single-unit neural recording with active microelectrode arrays," IEEE Trans. Biomed. Eng., vol. 48, Aug. 2001.

[4] R. A. Andersen and C. A. Buneo, "Intention maps in posterior parietal cortex," Аnnu. Rev. Neuroscience, vol. 25, pp. 189-220, 2002.
[5] D. Meeker, S. Cao, J. W. Burdick, and R. A. Andersen, "Rapid plasticity in the parietal reach region with a brain-computer interface," Soc. Neuroscience Abstracts, no. 357.7, 2002.

[6] [Online]. Available: http://www.bionictech.com/graphics/array.jpg

[7] P. K. Campbell, K. E. Jones, R. J. Huber, K. W. Horch, and R. A. Norman, "A silicon-based, three-dimensional neural interface: Manufacturing processes for an intracortical electrode array," IEEE Trans. Biomed. Eng., vol. 38, Aug. 1991.

[8] L. D. Castillo, R. Graber, S. D'Agostino, M. Mojarradi, and A. Mottiwala, "Flip chip packaging of a MEMS neuro-prosthetic system," in Int. Conf. Advanced Packaging Systems (Sponsored by IMAPS), Reno, NV, Mar. 10-13, 2002.

Mohammad Mojarradi (M'92) received the Ph.D. degree in electrical engineering from the University of California, Los Angeles (UCLA) in 1986.

Prior to joining Jet Propulsion Laboratory, Pasadena, CA, he was an Associate Professor at Washington State University and the Manager of the mixed-voltage/specialty integrated circuit group at the Xerox Microelectronics Center, El Segundo, CA. He is a Specialist in integrated mixed-signal/mixed-voltage electronic sensors, micromachined interface circuits, and mixed-mode integrated circuit design. He has more than 20 years of combined industrial and academic experience in his field. His current work focuses on developing highly efficient integrated mixed-signal electronics for sensors, actuators, and power management and distribution systems (PMAD) for avionics system on a chip for deep space using the SOI CMOS process.

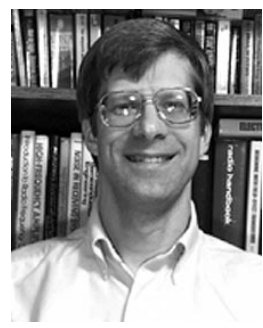

David Binkley (S'81-M'82-SM'93) received the B.S., M.S., and Ph.D. degrees in electrical engineering from the University of Tennessee, Knoxville.

He was formerly a cofounder and Vice President of integrated circuit development at Concorde Microsystems and a Senior Scientist at CTI/Siemens PET Systems, where he designed both discrete and integrated CMOS electronics for positron emission tomography (PET) medical imaging systems. In 2000, he joined the Electrical and Computer Engineering Department, University of North Carolina at Charlotte, as an Associate Professor. $\mathrm{He}$ and his students are researching analog design and testing methodologies, including micropower, low-noise analog CMOS design for neural implants, and radiation-hardened deep space applications. He is the author of more than 50 papers in analog circuit design and instrumentation, and he holds two U.S. patents. 


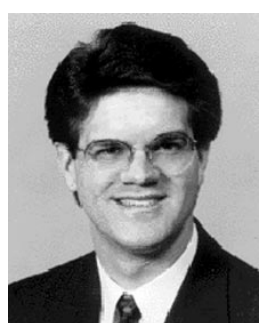

Benjamin Blalock (S'87-M'90) received the B.S. degree in electrical engineering from the University of Tennessee, Knoxville, in 1991 and the M.S. and $\mathrm{Ph} . \mathrm{D}$. degrees in electrical engineering from the Georgia Institute of Technology (Georgia Tech), Atlanta, in 1993 and 1996, respectively. Dr. P. E. Allen was his Ph.D. advisor.

He worked as an Analog IC Design consultant for Cypress Semiconductor Corp. and Concorde Microsystems, Inc. He first joined academia as an Assistant Professor in late 1996 with the Department of Electrical and Computer Engineering at Mississippi State University, Mississippi, MS, and then joined the Department of Electrical and Computer Engineering (ECE) at the University of Tennessee (UT), Knoxville, in 2001. He now leads the INSYTE research group at UT. He has more than 25 publications in the field of analog IC design and has contributed to The Circuits and Filters Handbook. At Georgia Tech, his research activities included noise characterization of GaAs MESFETs, BiCMOS operational amplifier design, CMOS current-feedback amplifier design, and ultralow-voltage CMOS analog circuit design for signal processing and power management applications. His current research focus includes mixed-signal/mixed-voltage circuit design for systems-on-a-chip in SOI technology, analog IC design for extreme environments, multigate transistors and circuits on SOI, body-driven circuit techniques for ultralow-voltage analog, and biomicroelectronics.

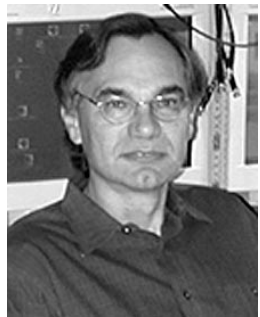

Richard Andersen is the James G. Boswell Professor of Neuroscience at the California Institute of Technology (Caltech), Pasadena. Since 1994, he has also been the Director of the Sloan Center for Theoretical Neurobiology at Caltech. He studies the neurobiological underpinnings to such brain processes as sight, hearing, and action planning. His lab is also studying neural prosthetics-electronic interfaces with the brain-which may some day allow disabled humans to operate external devices just by thinking.

Prof. Andersen is a Fellow of the American Association for the Advancement of Science and a Member of the American Academy of Arts and Sciences.

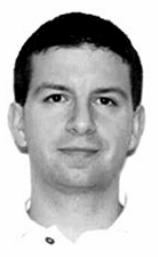

Norbert Ulshoefer (S'00-M'03) was born in Weilheim, Germany, in 1974. He received the Dipl.Ing. (FH) degree in electrical engineering from the University of Applied Sciences of Munich, Germany, in 1994, and the M.S. degree in electrical engineering at the University of North Carolina, Charlotte, in 2002.

From 2000 to 2002, he worked as a Research Assistant in the electrical and computer engineering department studies at the University of North Carolina, where his activities focused on low-noise micropower CMOS preamplifier designs for intracortical electrode arrays. He joined Infineon Technologies AG, Germany, where he has been engaged in analog and mixed-signal design of automotive power integrated circuits.

Travis Johnson (S'95-M'99) received the B.S.E. degree from Walla Walla College, College Place, WA, in 1995 and the M.S. degree in electrical engineering from Washington State University, Pullman, WA, in 1998.

From 1998 to 2001, he was at IMP working on power management ICs. He joined Jet Propulsion Laboratory, Pasadena, CA, in 2001 and is currently working on low-power analog front ends.

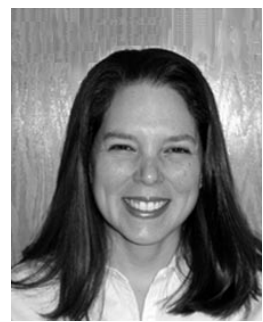

Linda Del Castillo received the B.S. degree in mechanical engineering from California State Polytechnic University, Pomona, and the M.S. and $\mathrm{Ph} . \mathrm{D}$. degrees in materials science and engineering from the University of California, Irvine.

She joined the Electronic Packaging and Fabrication Section at NASA's Jet Propulsion Laboratory, Pasadena, CA, in 2000. Since that time, she has worked in a variety of research areas, including chip-on-flex for space-based radar membrane antennas, electronic packaging for extreme environments, lead-free solder studies, embedded passives integration, MEMS fabrication (microfluidics and microinductors), and most pertinent to the present work, MEMS packaging of neuroprosthetic devices. 